\title{
Superluminal apparent velocities of relativistic electron beams in the solar corona
}

\author{
A. Klassen ${ }^{1}$, M. Karlický², and G. Mann ${ }^{1}$ \\ 1 Astrophysikalisches Institut Potsdam, An der Sternwarte 16, 14482 Potsdam, Germany \\ 2 Astronomical Institute of the Academy of Sciences of the Czech Republic, 25165 Ondřejov, Czech Republic
}

Received 7 May 2003 / Accepted 16 July 2003

\begin{abstract}
We present spectral and imaging observations of high frequency type III bursts appearing in pairs: a primary fast drifting component and a secondary "normal" drifting component. The primary bursts have generally higher frequency drift and start at higher frequencies. They show superluminal velocities up to $2.5 c$ ( $c$, speed of light), while the secondary component shows the usual $<0.5 c$ velocity expected for type III burst exciters. These superluminal velocities are explained as apparent velocities of relativistic electron beams propagating nearly along the line of sight towards the observer with velocities close to the speed of light. A model of type III burst pairs consisting of subsequent fast drifting and "normal" drifting components is presented.
\end{abstract}

Key words. plasmas - Sun: corona - Sun: particle emission - Sun: radio radiation

\section{Introduction}

Type III solar radio bursts represent electron beams propagating through the solar corona and/or interplanetary space and were first reported by Wild (1950). By propagating along open magnetic fields with a speed of about $0.3 c$ they generate high frequency plasma waves (Langmuir waves) which then can be transformed into electromagnetic waves at the plasma frequency $f_{\mathrm{p}}$ and its harmonic $2 f_{\mathrm{p}}$. Due to decreasing electron density and plasma frequency with distance from the Sun, the type III bursts in dynamic radio spectra show a rapid drift from higher to lower frequencies with a drift rate $D_{f}=\mathrm{d} f / \mathrm{d} t$ of about $100 \mathrm{MHz} \mathrm{s}^{-1}$ in the meter wave range. The frequency drift rate decreases with decreasing frequency and can statisticaly be approximated in meter range as $D_{f}=-0.01 \cdot f^{1.84}$ (Alvarez \& Haddock 1973) or as $D_{f}=-0.007 \cdot f^{1.76}$ (Mann et al. 1999). In the dm-range this relationship is given as $D_{f}=-0.09 \cdot f^{1.35}$ (Melendez et al. 1999). The corresponding range of speeds of the exciting electrons is about $0.1-0.6 c$.

Usually the type III bursts occur at frequencies below $300 \mathrm{MHz}$ and often they can be followed down to the lower frequency limit of space-based observations of about $20 \mathrm{kHz}$ if the spacecraft is located near the Earth. The high frequency type III bursts starting near or above $500 \mathrm{MHz}$ usually have a low frequency cutoff near $100 \mathrm{MHz}$ and do not escape into interplanetary space. It was found that they tend to appear in pairs. Bursts in each pair have considerably different properties. The primary burst has a generally higher frequency drift and starts at higher frequencies. The second burst is a

Send offprint requests to: A. Klassen, e-mail: aklassen@aip.de "normal" type III burst with slower drift (Benz et al. 1982; Poquérusse 1994). We will use the same definition of such pairs as Poquérusse (1994): the primary fast drifting component as type IIId burst and the second component as a "normal" type IIIn burst. A scheme of such type IIId + IIIn fundamental and harmonic pairs is shown at Fig. 1.

Poquérusse (1994) presented evidence of relativistic electron beams generating type IIId bursts in the solar corona with fast drift rates of $D_{f} \simeq 1500 \mathrm{MHz} \mathrm{s}{ }^{-1}$ in the range 500$100 \mathrm{MHz}$. Using a density model with density scale height of $H=10^{5} \mathrm{~km}$ and the drift rates of type IIId he interpreted these as due to generation of plasma waves by the usual bump-on-tail instability at the harmonic mode by relativistic electron beams with speeds close to the speed of light. It was proposed that such relativistic type IIId bursts can result in superluminous apparent speeds if the beam moves towards the observer.

Very fast transverse speeds in the corona were already deduced from radioheliographic observations by Raoult et al. (1989). Comparing type III burst positions on the solar disc with the flare position led these authors to conclude that some type III bursts had speeds larger than $0.6 c$.

Low speeds in the interplanetary medium were obtained by Dulk et al. (1987). The authors used a detailed method involving in situ detection convincingly showing that type III burst speeds at low frequencies are of the order of $0.1 c$, much lower than in the corona.

Here we report observations of pairs of type III bursts that start at high frequencies, $\leq 1900 \mathrm{MHz}$. Some of them propagate through the corona and interplanetary space and are followed down to a frequency of $20 \mathrm{kHz}$ (i.e. about $1 \mathrm{AU}$ ). 


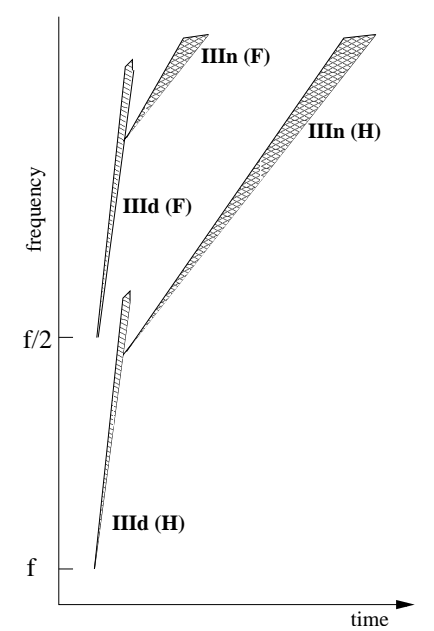

Fig. 1. Scheme of type III pairs: IIId-IIIn bursts. Both fundamental and harmonic emission are shown. Compare with the bursts on 25 September 1997 (Fig. 5).

We use spectral and heliographic observations to determine the speeds and trajectories of the exciting electrons. The observed burst pairs, which are likely to be the type IIId-IIIn bursts reported by Poquérusse (1994), show in their high-frequency parts very fast drifts and relativistic velocities. Using Nançay radio imaging observations we will show that such relativistic bursts lead to superluminal apparent speeds of radio sources. It is the same effect as observed in the case of extragalactic radio jets (Pearson \& Zensus 1987).

\section{Instruments}

The radio spectrum in the range $2000-0.020 \mathrm{MHz}$ was observed by the AO Ondrejov spectrograph (2000$800 \mathrm{MHz}$, time resolution $0.1 \mathrm{~s}$ ), radio spectrometer of Astrophysikalisches Institute Potsdam (800-40 MHz, time resolution $0.1 \mathrm{~s}$ ) and by the space-based WAVES instrument (14-0.020 MHz, time resolution $60 \mathrm{~s}$ ) on board the Wind spacecraft (Jiřička et al. 1993; Mann et al. 1992; Bougeret et al. 1995). The imaging observations were made by the Nançay Radioheliograph (henceforth NRH, Kerdraon \& Delouis 1997) at five frequencies 432, 410, 327, 236, and $164 \mathrm{MHz}$ (time resolution $0.12 \mathrm{~s}$ ).

\section{Observations}

\subsection{Bursts on 31 August 1998}

On 31 August 1998 three high frequency type III bursts were observed. They belong to a group of type III bursts associated with a M1.5/SF flare (start at 1529 UT, peak at 1539 UT) in AR 8307 at co-ordinates N32W69. Figure 2 presents the dynamic radio spectrum of the bursts in the range 2000$0.010 \mathrm{MHz}$. All bursts are structureless, meaning only one harmonic is observed. The degree of polarization is low $(\leq 10 \%)$ in the range 432-164 MHz. Both these facts, together with the fact that the bursts occur near the solar limb indicate the

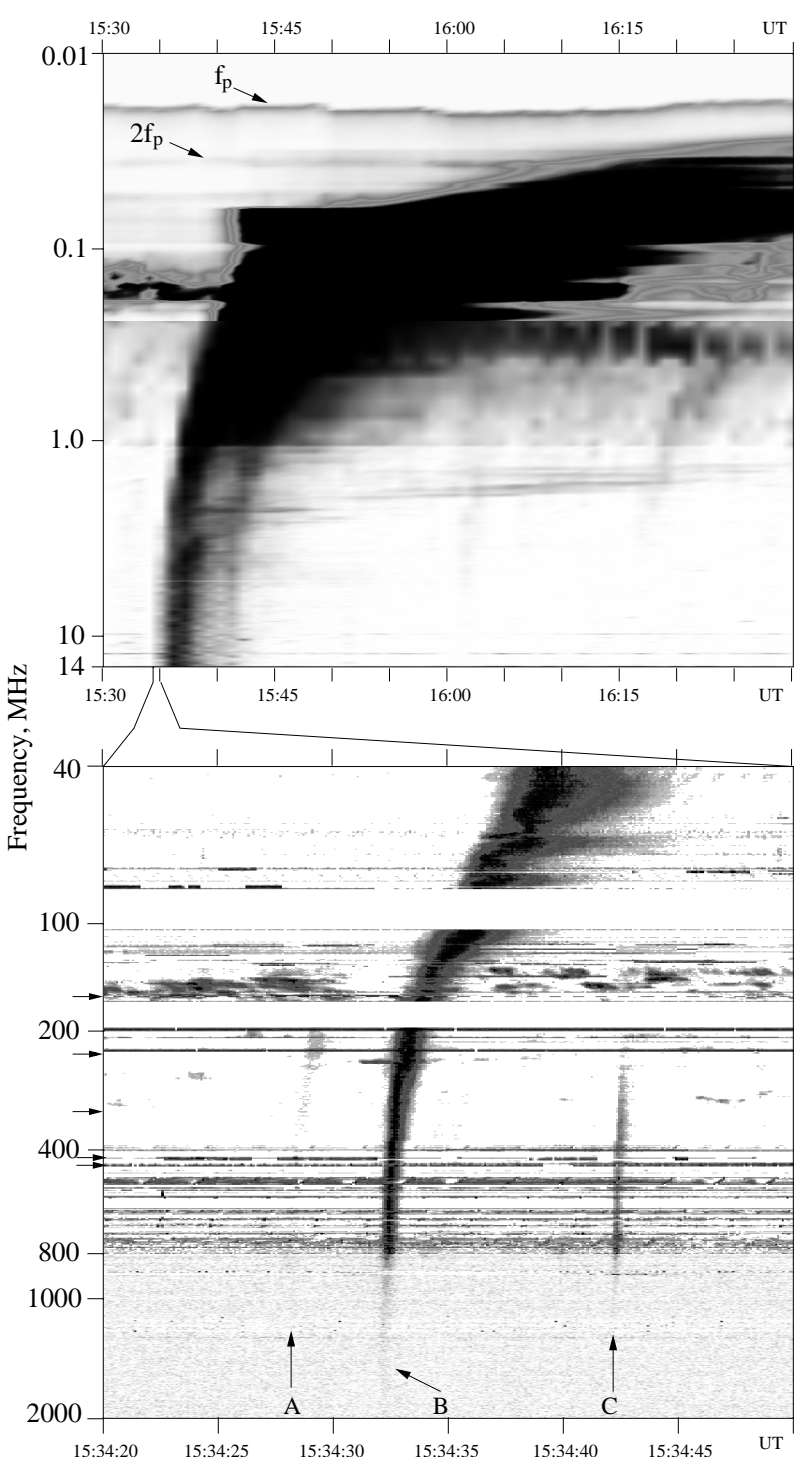

Fig. 2. Dynamic radio spectrum of the event on 31 August 1998, in the range 2000-0.010 MHz (Ondrejov, Tremsdorf, Wind/WAVES radiospectrographs). Bottom panel: Three type III bursts (A, B, C) in the range 2000-40 MHz. At the left axis the arrows point at the $\mathrm{NRH}$ frequencies. At these frequencies the source positions are obtained. Only one burst (B) moves across the corona and escapes into IP medium. In the 14-0.040 MHz range this burst (B) merges with following bursts to one IP type III burst. Top panel: The IP type III burst drifts until $0.030 \mathrm{MHz}(30 \mathrm{kHz})$. It is stopped between the local fundamental $f_{\mathrm{p}}$ and harmonic $2 f_{\mathrm{p}}$ plasma level of the Wind spacecraft.

emission at the harmonic mode $2 f_{\mathrm{p}}$, because the emission at the fundamental mode could be strongly absorbed (e.g. Suzuki \& Dulk 1985). Figure 3 presents the time profiles of one type III burst at 15:34:33 UT at four NRH frequencies.

The first burst (labeled as A) is observed in the 1000 $150 \mathrm{MHz}$ range, the second (B) at $1900-0.040 \mathrm{MHz}$, and the third (C) at 1300-200 MHz. Only one burst (B) moves out of the corona into the interplanetary space (IP). Here it merges together with other individual type III bursts associated with the impulsive flare phase and is visible down to about $0.020 \mathrm{MHz}$, i.e. close to the local plasma frequency $\left(f_{\mathrm{p}}\right.$ or $2 f_{\mathrm{p}}$, Fig. 2) 


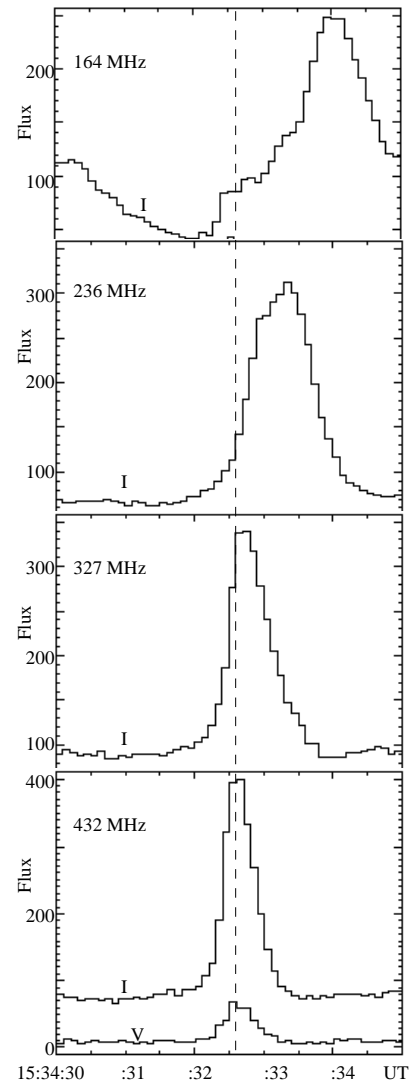

Fig. 3. Time profiles of the type III burst "B" on 31 August 1998 in the range $432-164 \mathrm{MHz}$. I-intensity, $v$-polarisation.

near the Wind spacecraft (1 AU). It seems that the corresponding electron beam does not affect the spacecraft, because the plasma wave detector (TNR receiver, range 4-256 kHz) onboard Wind does not show any reaction, i.e. expected Langmuir waves from the arrived suprathermal electrons. That means that the spacecraft and the beam site were not magnetically connected.

The other two bursts show strict frequency cutoffs at 150 and $200 \mathrm{MHz}$, respectively. That means that the corresponding beams were stopped or the generation of the radio emission ceased. In each case the radio intensity went below the instrument sensivity threshold.

Another important feature of the burst (B) is its splitting into two bursts during its propagation from high to low frequencies. While the new (secondary) burst escapes from the primary component in the range $300-250 \mathrm{MHz}$, the primary one drifts somewhat further practically without any change in $D_{f}$ and disappears rapidly.

The difference of the drift rates of both components is very large. The primary burst drifts at a rate of $D_{f} \geq 1000 \mathrm{MHz} \mathrm{s}^{-1}$ at $432-327 \mathrm{MHz}$ whereas the drift rate of the secondary component is about $40 \mathrm{MHz} \mathrm{s}^{-1}$ at $220-110 \mathrm{MHz}$. While burst (A) is weak and shows the same tendency to split, the burst (C) does not split and disappears near $200 \mathrm{MHz}$. The difference between bursts (B) and (C) is that the first one displays both components (IIId+IIIn) whereas the second one the IIId component only. The main parameters of all bursts are given in Table 1 .
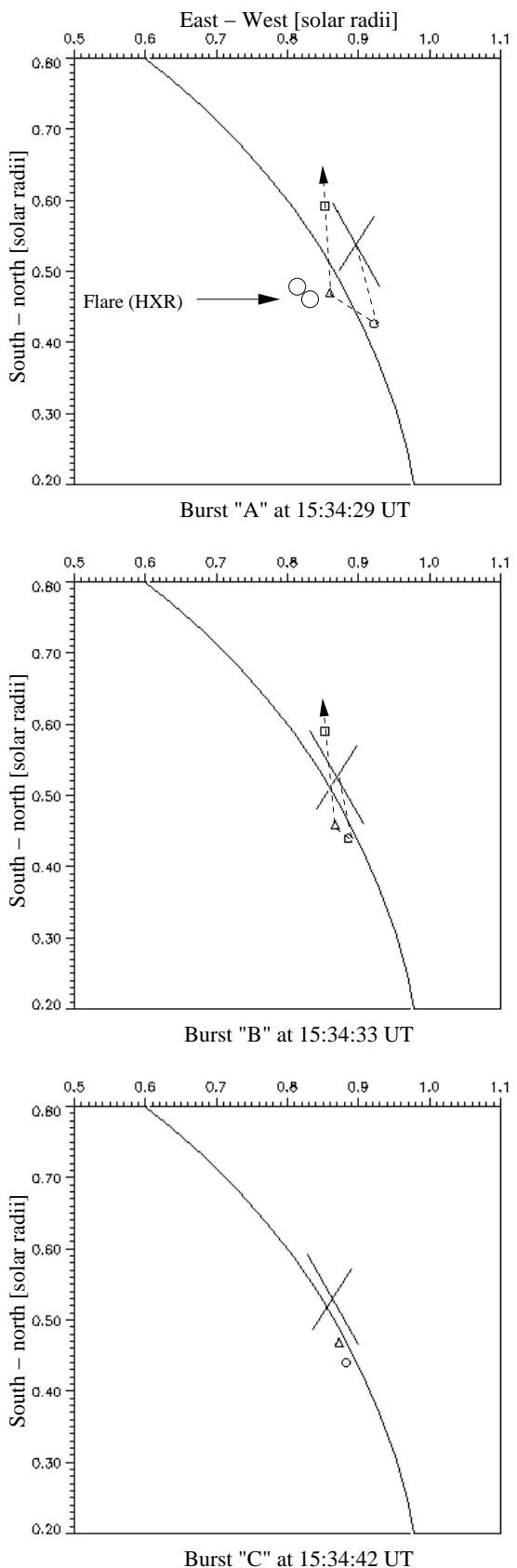

Fig. 4. Positions of radio source centroids (NRH) of type IIIs on 31 August 1998: type IIId at 432 (cross, source half power width) and $327 \mathrm{MHz}$ (circle), type IIIn at 236 (triangle), and at $164 \mathrm{MHz}$ (square). Flare (HXR) - the position of the hard X-ray footpoint sources.

The drift rates (see Table 1) of observed type IIIs are close to the empiric relations of Alvarez \& Haddock (1973) and Mann et al. (1999).

Figure 4 presents type III source positions and their trajectories at four NRH frequencies. The burst trajectories show a strong non-radial propagation. At first the bursts intersect the $432 \mathrm{MHz}$ of NRH and the sources occur near and almost in a radial direction above the flare and HXR site. If we take as an example the burst (B) at 15:34:32 UT we see that at successively lower frequencies $(432,410 \& 327 \mathrm{MHz})$ the sources 
are displaced to the south in direction nearly perpendicular to the radial direction. Note the source at $410 \mathrm{MHz}$ is not shown, because close to the source at $432 \mathrm{MHz}$. At 236 and $164 \mathrm{MHz}$ the sources present an unexpected behavior: they are moving back in the opposite direction. Taking into account the spectral behaviour of the burst (B), especially the burst splitting near $250 \mathrm{MHz}$, we suppose that the sources at 432, 410, 327, and partly at $236 \mathrm{MHz}$ belong to the fast drifting type IIId burst, whereas the sources at 236 and $164 \mathrm{MHz}$ to the "normal" drifting type IIIn one.

Such a peculiar trajectory of type III sources in projection on the plane of the sky suggests that the exciting electron beam moves directly to the observer at small angles and along a curved trajectory. The other two bursts (A \& B) show similar trajectories with small variations.

It should be noted that to reduce the coronal propagation effect - the refraction of burst radiation due to the density inhomogeneities in the corona, all radio source positions were measured at the second harmonic mode, $2 f_{\mathrm{p}}$, only. Because this effect is relatively strong for the fundamental emission, it could lead to the displacement of the observed source position in comparison to the real source. To determine the $v_{\mathrm{app}}^{432-327}$ (see Sect. 3.4) we use the relative distance $\left(\Delta L_{432-327}\right)$ between source positions at two frequencies, and not the absolute positions. Furthermore, the refraction effect should shift the radio sources in the same direction. Zlotnik et al. (1998) found also that the harmonic $\left(2 f_{\mathrm{p}}\right)$ and the second harmonic $\left(3 f_{\mathrm{p}}\right)$ sources at least for type II bursts are cospatial. Therefore we suppose that propagation effects do not significantly alter the positions of the harmonic type III emission and do not result in appearence of "apparent sources" leading to a significant error in the determination of $\Delta L_{432-327}$ and $v_{\text {app }}^{432-327}$.

\subsection{Burst on 7 August 1998}

The harmonic type IIId-IIIn pair is shown in Fig. 5 (top). The fundamental and the harmonic modes of type IIId are visible at 700-200 and 250-110 MHz, respectively. While the harmonic component IIIn is very weak, the fundamental component shows a strong emission at $85-40 \mathrm{MHz}$.

This burst group was associated with a small $\mathrm{H}_{\alpha}$ flare (SF) and ejecta in AR 8293 (S20E05) as clearly seen in the TRACE image. Figure 6 shows the location of the type III burst sources superposed on the TRACE image obtained at the same time, at 15:16:51 UT.

The main points deduced from Fig. 6 are: (a) there is a very good spatial coincidence between the type III position at $432 \mathrm{MHz}$ and the ejecta at $171 \AA$ (TRACE, 15:16:51 UT); (b) the burst at $432 \mathrm{MHz}, 410 \mathrm{MHz}$ (not shown, close to the source at $432 \mathrm{MHz}$ ), and $327 \mathrm{MHz}$ is located along and at the border of two EUV loop systems. The direction of the EUV ejecta and of the radio source movement from 432 to $327 \mathrm{MHz}$ is the same; (c) from 236 to $164 \mathrm{MHz}$ the type IIIn burst moves in a direction nearly perpendicular to the previous one.
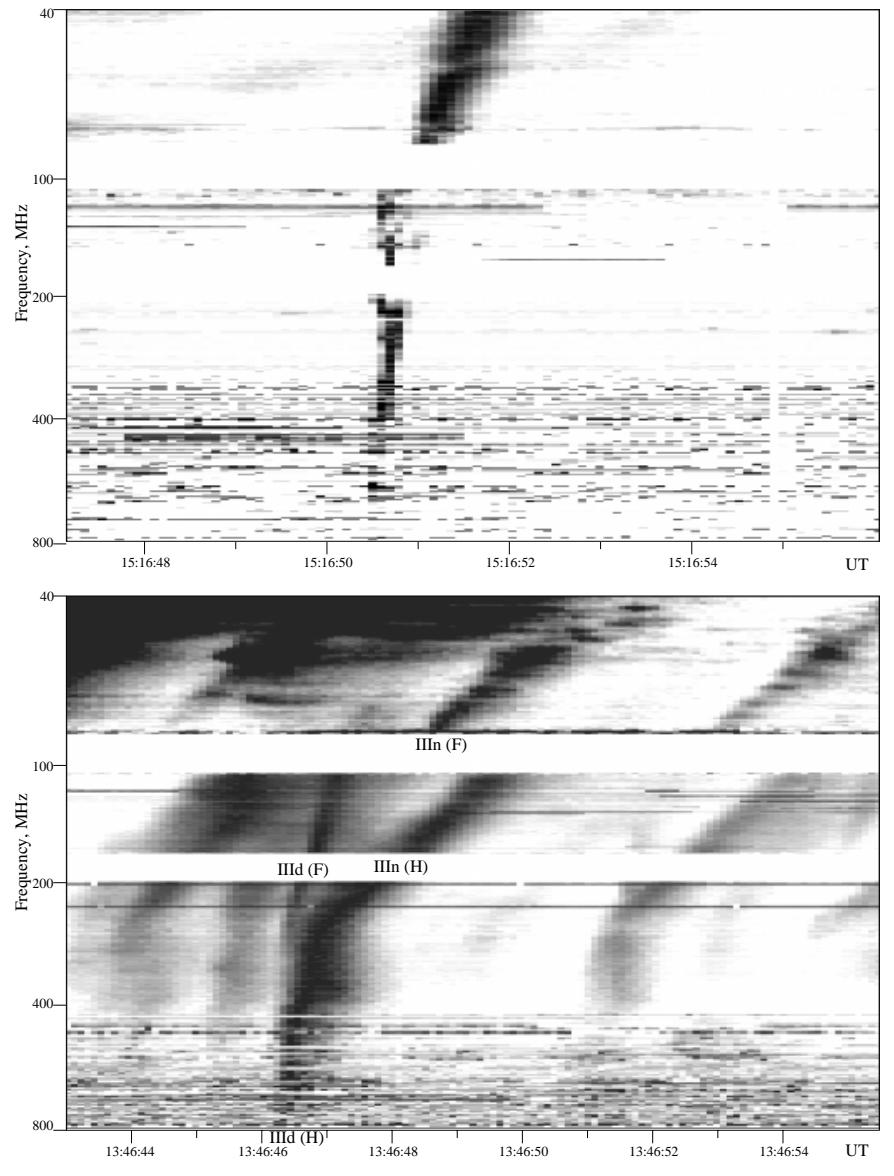

Fig. 5. Type IIId+IIIn bursts on 7 August 1998 (top) and on 25 September 1997 (bottom).

\subsection{Burst on 25 September 1997}

Figure 5 (bottom) show pairs of type IIId-IIIn bursts. The burst at 13:46:47 UT is the most intense, contains both components (IIId \& IIIn), and they both display a fundamental-harmonic structure. This group of bursts was associated with activity on the solar disc (AR 8088, S28E08). The harmonic of the IIId and IIIn bursts appear in the range of 700-250 MHz, and 300$100 \mathrm{MHz}$, respectively. Thus, it was possible to derive the positions of both components at all NRH frequencies. Figure 6 (bottom) shows the positions of both components at 13:46:47 UT. The dispersion of the type IIId burst position with frequency at 432, 410, $327 \mathrm{MHz}$ is SW - directed along a straight line. The positions of the type IIIn burst at 236 and $164 \mathrm{MHz}$ are located almost along a line in an antiparallel NE - direction.

\subsection{Speed of type III burst exciters in corona}

We suppose that type III bursts are tracers of propagating electron beams. If we take this into account we can derive the speed of electron beams producing type III bursts directly from positions of radio sources at different frequencies. Because the fast drifting component IIId is definitely observed at 432,410 \& $327 \mathrm{MHz}$, we use the source positions and the time if the burst reaches its emission maximum at $432 \& 327 \mathrm{MHz}$ to get the distance between these sources in the plane of the sky $\Delta L_{432-327}$ 

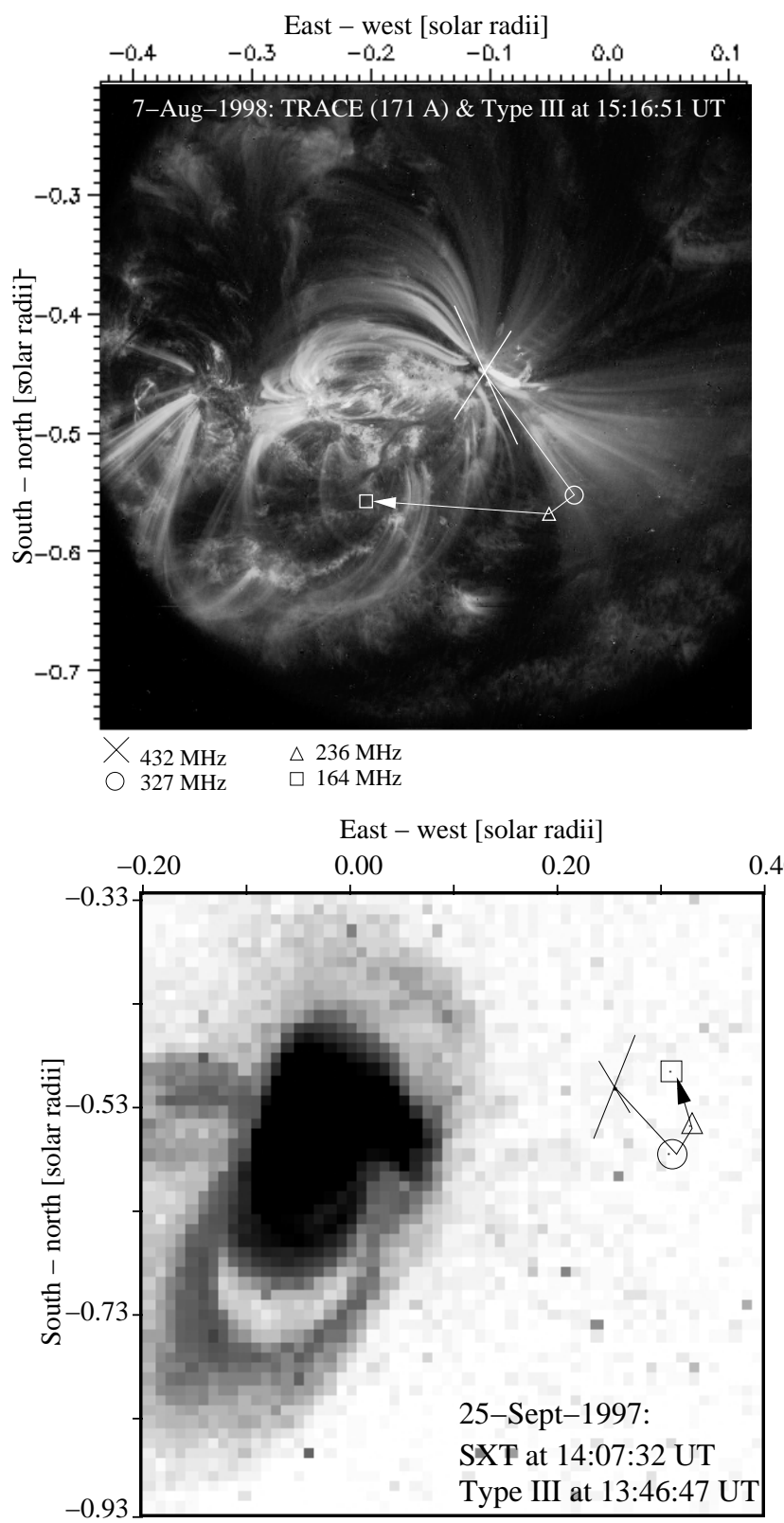

Fig. 6. NRH positions of type IIId+IIIn sources on 7 August 1998 (top) and on 25 September 1997 (bottom), overlaid on TRACE (171 A,, 15:16:51 UT) and Yohkoh SXT (14:07:32 UT) images, respectively. The IIId bursts are presented at 432 (cross, source half power width) and $327 \mathrm{MHz}$ (circle). The IIIn bursts at 236 (triangle) and $164 \mathrm{MHz}$ (square).

and the time delay $\Delta t_{432-327}$ (Table 1 ). The source parameters at $410 \mathrm{MHz}$ and $432 \mathrm{MHz}$ are very similar, therefore we use the observations at $410 \mathrm{MHz}$ to control the parameters only. The apparent transversal speed of type III exciters is then:

$v_{\text {app }}^{432-327}=\Delta L_{432-327} / \Delta t_{432-327}$.

To get the apparent speed of exciters of the second component (IIIn) we use the source parameters at 236 and $164 \mathrm{MHz}$. Table 1 gives the speeds of type IIId and IIIn components (Apparent transversal speed).

The speed of type III burst exciters can be estimated by another method using a density model and observed frequency drift rates $\left(D_{f}\right)$. The exciter speed is related to the frequency drift by

$V_{\mathrm{drift}}=\frac{2 H D_{f}}{f \cos \alpha}$

where $\alpha$ is the angle between the beam direction and the radial direction, and $H$ is the the hydrostatic scale height,

$H=\frac{k_{\mathrm{b}} T}{\mu m_{\mathrm{p}} g_{\odot}}$

where $k_{\mathrm{b}}$ is the Boltzmann's constant, $T$ electron temperature for an isothermal structure, $\mu \simeq 0.6$ is the mean molecular weight, $m_{\mathrm{p}}$ the proton mass, and $g_{\odot}$ the acceleration of gravity at the Sun. For the coronal conditions and $T=2 \mathrm{MK}$, $H \simeq 10^{5} \mathrm{~km}$. Similar scale heights were found for EUV loops in active regions (ARs) (Aschwanden et al. 2001). Taking $H=10^{5} \mathrm{~km}$ and the $D_{f}$ measured in the range $432-327 \mathrm{MHz}$ we estimate the radial component of the type III exciter speeds $V_{\text {drift }}=200000-525000 \mathrm{~km} \mathrm{~s}^{-1}$ (see the "apparent radial speed $\left(D_{f}\right)$ " in Table 1). It is assumed that the beams propagate along the density gradient which average corresponds to the radial direction (i.e. the $\alpha \simeq 0^{0}, \cos \alpha \simeq 1$ ).

It was found that the coronal streamers and ARs have a scale height that exceeds the hydrostatic scale height by factor of up to 2.3 (Aschwanden et al. 2001). Furthermore, at least the bursts on 31 August 1998 show a strongly not radial propagation. Both facts show that the velocities estimated from the spectrum using $D_{f}$ are rather a low limit for $V_{\text {drift }}$. Nevertheless they are comparable with velocities obtained directly from radio imaging observations and confirm the superluminal character of type IIId bursts.

Note, that the apparent transversal speed $v_{\text {app }}^{432-327}$ determined from positional measurement has a different sense than that determined using frequency drift $V_{\text {drift }}$. The first method gives the component of the speed in the plane of the sky, i.e. perpendicular or transverse to the observer's direction. The second method gives the component of the speed along the density gradient.

In general, the type IIId burst time delays $\Delta t_{432-327}$ between the high and the low frequencies are comparable with the time resolution of the instruments used. Therefore, for a more quantitative study of type IIId bursts a time resolution better than $0.1 \mathrm{~s}$ is desirable.

\subsection{Speed of type III burst exciters in IP medium}

The electrons of burst (B) on 31 August 1998 were injected at $(15: 34.5-500 \mathrm{~s})$ UT, they propagate through the corona and IP medium and arrive the local plasma level $\left(2 f_{\mathrm{p}}\right)$ at about 16:30 UT (Fig. 2). If we assume that the electrons move directly to the spacecraft, then we can estimate the speed of electrons as $V_{\mathrm{e}}=L / t_{\text {(sun-earth) }}$, where $L \simeq 1.2 \mathrm{AU}$ is the length of the path along the magnetic field spiral from the Sun to the spacecraft, $t_{\text {sun-earth }}$ is the flight time of electrons. We get $V_{\mathrm{e}} \simeq$ $45000 \mathrm{~km} \mathrm{~s}^{-1}$, i.e. $0.15 c$. 
Table 1. Type III burst parameters.

\begin{tabular}{|c|c|c|c|c|c|}
\hline \multirow[b]{2}{*}{ Parameter } & \multicolumn{3}{|c|}{31 August 1998} & \multirow[t]{2}{*}{ 7-Aug.-1998 } & \multirow[t]{2}{*}{ 25-Sep.-1997 } \\
\hline & $\begin{array}{c}\text { Burst (A), } \\
\text { 15:34:29 UT }\end{array}$ & $\begin{array}{c}\text { Burst (B), } \\
\text { 15:34:32 UT }\end{array}$ & $\begin{array}{c}\text { Burst (C), } \\
\text { 15:34:43 UT }\end{array}$ & & \\
\hline Range, $\mathrm{MHz}$ & $1000-150$ & $1900-0.040$ & $1300-200$ & $700-40 X$ & $700-40 X$ \\
\hline \multicolumn{6}{|l|}{ Intrinsic duration, $s$} \\
\hline $800-400 \mathrm{MHz}$ & $\simeq 0.5$ & $0.45-0.6$ & $0.3-0.4$ & $0.1-0.2$ & $0.6-0.7$ \\
\hline $400-200 \mathrm{MHz}$ & 0.75 & $0.6-1.2$ & $0.5-0.6$ & $0.2-0.3$ & $0.7-0.9$ \\
\hline \multicolumn{6}{|l|}{ Drift rate, $\mathrm{MHz} \mathrm{s}^{-1}$} \\
\hline $800-400 \mathrm{MHz}$ & $\simeq 2000$ & 3000 & $\geq 4000$ & $\geq 4000$ & 1400 \\
\hline $432-327 \mathrm{MHz}$ & $350-400$ & $\geq 1000$ & $\geq 1000$ & $>1000$ & 500 \\
\hline \multicolumn{6}{|l|}{ Position shift, km } \\
\hline$\Delta L_{432-327}$ & 79000 & 61000 & 63000 & 92000 & 55000 \\
\hline$\Delta t_{432-327}, \mathrm{~s}$ & 0.24 & 0.12 & 0.12 & $\leq 0.12$ & 0.24 \\
\hline \multicolumn{6}{|c|}{ Apparent transversal speed (NRH) } \\
\hline$v_{\text {app }}^{432-327}, \mathrm{~km} \mathrm{~s}^{-1}($ IIId $)$ & 330000 & 510000 & 530000 & 770000 & 230000 \\
\hline$v_{\text {app }}^{236-164}, \mathrm{~km} \mathrm{~s}^{-1}($ IIIn $)$ & 106000 & 130000 & - & - & 40000 \\
\hline \multicolumn{6}{|c|}{ Apparent radial speed $\left(D_{f}\right)$} \\
\hline$V_{\mathrm{drift}}^{432-327}, \mathrm{~km} \mathrm{~s}^{-1}($ IIId $)$ & 200000 & $\geq 525000$ & $\geq 525000$ & $>525000$ & 260000 \\
\hline
\end{tabular}

All measurements are made at the harmonic mode: intrinsic duration at the bursts half intensity, the drift rate $D_{f}$ and the time delay $\left(\Delta t_{432-327}\right)$ at the maximum intensity from the dynamic spectrum and at corresponding NRH frequencies. X- means that the burst extends beyond instrument range.

\section{Observational results}

The investigation of fast drifting type III bursts combining spectrographic and imaging observations has revealed the following results:

1. Observed type III bursts tend to occur in pairs. The components in each pair show strongly different properties: a fast drifting type IIId and a "normal" drifting type IIIn component. Some of them represent only the IIId component, without IIIn and vice versa.

2. Both the spectrographic and the imaging observations suggests that the second component (IIIn) separates from the first one (IIId). It means that near the splitting frequency both components have a common source: the type IIIn burst escapes from the type IIId burst source.

3. The speed of type IIId burst exciters estimated independently by two different methods show superluminal apparent velocities of $(\leq 2.5 c)$. That means that the real speed of electron beams could be near to the speed of light, i.e. in the range of $220000-280000 \mathrm{~km} \mathrm{~s}^{-1}$. That corresponds to electron energies of $0.25-2.0 \mathrm{MeV}$.

4. Type IIIn - the second ("normal") component of the pairs shows speeds of $0.13-0.4 c$, which is usual for type III bursts. Those speeds correspond to electron energies of 4 $55 \mathrm{keV}$.

5. In the events presented here the trajectories of "normal" type IIIn bursts are not a simple straightforward extension of type IIId trajectories, but show a sudden change in direction of propagation.

\section{Model}

\subsection{Apparent superlumenal velocities}

It is assumed that an electron beam generates the observable radio emission along its trajectory (see Fig. 7). Due to the beam velocity $\left(v_{\text {real }}\right)$, the beam propagation time $t_{0}$ between two positions ( $\mathrm{a}$ and $\mathrm{b}$ ) is related to the time difference $(\Delta t)$ between observations of the radio source in projection at ( $a$ and $b$ ) by:

$\Delta t=t_{0}\left(1-\frac{v_{\text {real }}}{c} \cos \phi\right)$,

where $\phi$ is the angle between the direction of the beam propagation and the direction to an observer, and $c$ is the light speed. The apparent velocity of the radio source is expressed as:

$v_{\text {app }}=\frac{\Delta L}{\Delta t}$,

where $\Delta L$ is the distance between the points a and b in projection on the sky (Fig. 7). But we can express this distance also as

$\Delta L=t_{0} v_{\text {real }} \sin \phi$.

Thus the apparent transversal velocity of the radio source can be calculated by

$v_{\text {app }}=\frac{v_{\text {real }} \sin \phi}{1-\left(v_{\text {real }} / c\right) \cos \phi}$.

Then the real velocity of the radio source is given by

$v_{\text {real }}=\frac{c v_{\text {app }}}{c \sin \phi+v_{\text {app }} \cos \phi}$. 


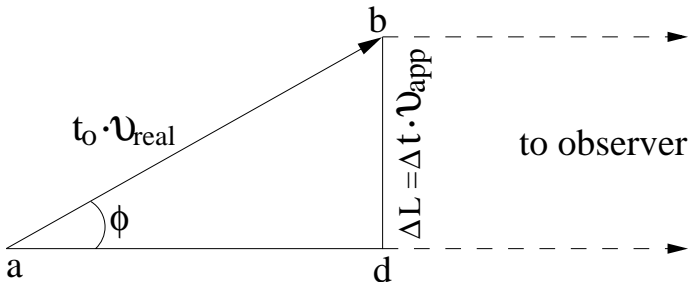

Fig. 7. Geometry of an observation of the electron beam propagation. The beam propagates from point a to $\mathrm{b}$ during a time $t_{0}, v_{\text {real }}$ is the real electron beam velocity, $\Delta t$ is the measured time difference between the beam observation at the positions a and $\mathrm{b}, v_{\text {app }}$ is the observed apparent velocity of the beam, and $\phi$ is the angle between a direction of the beam and that to an observer.

We expressed the apparent velocities for several real velocities of the beam depending on the view angle in Fig. 8. As it can be seen for the relativistic electron beams with velocities close to the speed of light, this apparent velocity can be superluminal. This approach is similar to that for extragalactic radio jets (Pearson \& Zensus 1987; see also Poquérusse 1994).

Using the observed apparent superluminal velocities of type IIId bursts (Table 1) and assuming a view angle of $\phi=20^{\circ}$ we find that the real velocities are in the range $0.93 c-0.75 c$. This corresponds to electron energies of $2.0-0.25 \mathrm{MeV}$.

\subsection{Model of type IIId and IIIn bursts generated by one electron stream released with a power-law distribution function in acceleration space}

This model agrees with that of Poquérusse (1994). Let us assume that in the acceleration region, superthermal electrons are accelerated with a power-law distribution function. Furthermore, let the energy power-law index $b$ for the relativistic part of electrons be smaller than $3\left(f \sim\left(E_{0} / E\right)^{b}\right.$, where $f$ is the distribution function and $E$ is the electron energy). As shown by Poquérusse (1994), due to the accumulation of electrons (in velocity space) close to the speed of light a very narrow beam with small velocity dispersion can be formed (the condition for MHD instability stage (or reactive stage, e.g. Melrose 1980) of the two-stream instability can be fulfilled), and a type IIId burst is generated from the acceleration source up to some coronal height (corresponding to about of $150 \mathrm{MHz}$ ), where the validity of the condition for the hydrodynamic instability is interrupted. This means that the starting frequency of this type IIId burst corresponds to the acceleration site and the density derived from the starting frequency is nearly the density in the acceleration region.

On the other hand, for lower velocities, the electron distribution function is also a power-law in the acceleration region, therefore there is no bump there and no instability initially. But this part of the distribution function evolves in space. We can express its evolution as follows (Karlický \& Krlín 1983)

$f(h, v, t)=\frac{n_{\mathrm{b}}(\delta-1)}{v_{0}}\left(\frac{v_{0}}{v}\right)^{\delta} \exp \left[-\alpha^{2}(h-v t)^{2}\right]$,

for $v>v_{0}$, where $f$ at the initial time $t=0$ is a power-law function with a power-law index $\delta$ (the corresponding energy

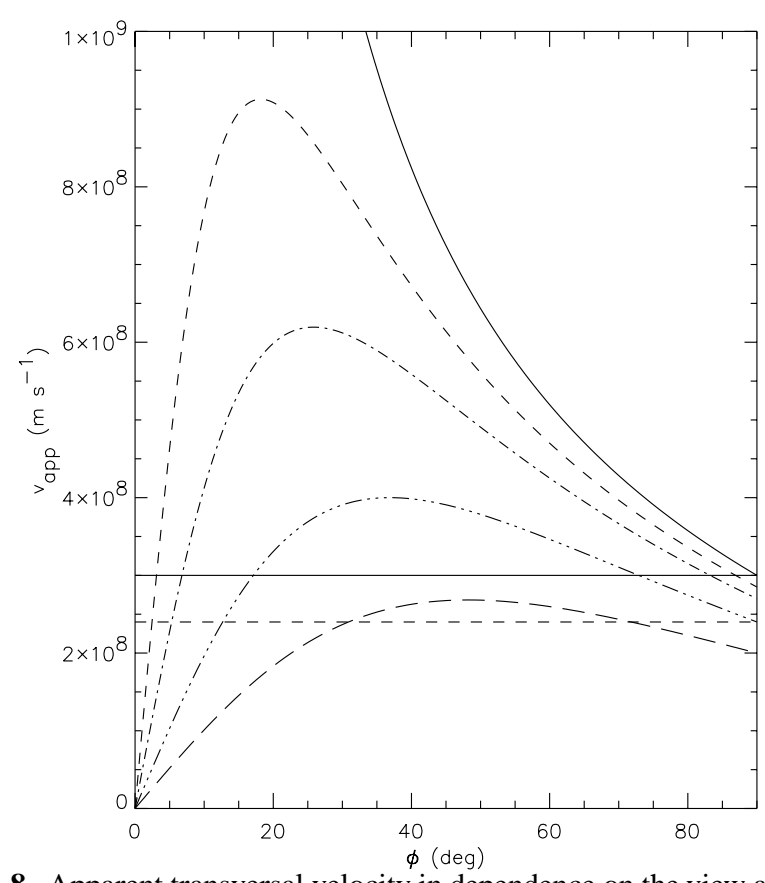

Fig. 8. Apparent transversal velocity in dependence on the view angle $\phi$ (see Fig. 7) for the beam with velocity $v=3 \times 10^{8} \mathrm{~m} \mathrm{~s}^{-1}$ (full line), $v=2.85 \times 10^{8} \mathrm{~m} \mathrm{~s}^{-1}$ (dashed line), $v=2.7 \times 10^{8} \mathrm{~m} \mathrm{~s}^{-1}$ (dotdashed line), $v=2.4 \times 10^{8} \mathrm{~m} \mathrm{~s}^{-1}$ (dot-dot-dot-dashed line), and $v=$ $2.0 \times 10^{8} \mathrm{~m} \mathrm{~s}^{-1}$ (long dashed line). The horizontal full line corresponds to the speed of light and the horizontal dashed line to the one of the observed velocity $230000 \mathrm{~km} \mathrm{~s}^{-1}$.

index $b=2 \times \delta$ can be higher than that in the relativistic part of the distribution function), $n_{\mathrm{b}}$ is the beam density, $v$ is the velocity, $\mathrm{h}$ is the coronal height, $v_{0}$ is the low velocity cut-off, $\alpha$ is the parameter expressing the width of the Gaussian form of the spatial distribution in the acceleration region at the initial time. As can be seen, during the evolution of this distribution function at some specific time and at some specific distance (at heights corresponding to about $150-200 \mathrm{MHz}$ ) from the acceleration site the bump-on-tail is formed (the growth rate of this instability ovecomes the collisional damping) and a normal type III burst starts. This part of the distribution function contains many more electrons than the high speed part, and can generate a type IIIn burst on a long trajectory, sometimes all the way from the solar flare to the Earth. Thus electrons of one distribution function can explain two different type III bursts: a) its relativistic part generates the high-frequency type IIId burst and b) its part with classical velocities the normal type IIIn burst, but after some evolution.

\section{Discussion and conclusion}

In the previous section we proposed a model explaining the subsequent type IIId and type IIIn bursts by two different emission regimes of the beam. The relativistic regime, generating the type IIId burst, operates very soon after the release of the beam and tends to be the most efficient at the beginning. But as fast electrons lose progressively their energy into Langmuir waves, at some point there are too few relativistic electrons left: the relativistic regime ceases and the type IIId burst disappears. 
On the contrary, the classical regime of the beam instability, generating normal type IIIn burst, starts slowly and tends to be more and more efficient as the "bump-on-tail" is formed by the propagation.

Although this model explains many characteristics of the pair of subsequent type IIId and IIIn bursts, one observational aspect still needs further careful consideration. Namely, the change of the type IIId burst into a type IIIn burst, the frequency stop of radiation of type IIId, and the sudden change of trajectories that were observed at about the same frequency range 150-200 MHz. In the present model this fact is considered to be by chance. But using the present observational data we cannot exclude some further possibilities:

1. The relativistic beam, generating the high-frequency fast drifting type IIId burst, is moving along a magnetic loop up to the top, where it penetrates into the bottom part of the current sheet expected in a streamer structure. The beam increases the local electrical resistivity, as suggested in the paper by Karlický \& Jungwirth (1989) and then the induced electric field accelerates the new electron beam which generates the normal type IIIn burst.

2. The relativistic beam generates strong electromagnetic radiation, which accelerates a new beam, generating the type IIIn burst, in the neighbouring open magnetic field structure. This idea is similar to that suggested by Sprangle \& Vlahos (1983).

3 . At the top of the closed magnetic field lines the relativistic beam penetrates into a zone of plasma waves as discussed by Karlický et al. (1996). Here the parameters of the beam change into these for a type IIIn burst.

4. There are two different beams propagating in two neighbouring but different magnetic loops as suggested by Benz et al. (1982). This model is less likely because at 236 and $327 \mathrm{MHz}$ where both components were simultaneously observed, they had a common source.

Our observations show that high frequency type III bursts can appear in pairs: a primary fast drifting high frequency component (IIId) and a secondary "normal" drifting component (IIIn). Together, they can cover a very broad spectral range of $2000-0.020 \mathrm{MHz}$, i.e. from the flare site to the Earth orbit (1 AU). Using direct imaging observations we found out for the first time that the primary component shows a superluminal velocity up to $2.5 c$, while the secondary component has the usual velocity of type III burst exciters $(<0.5 c)$. The superluminal apparent velocities of type IIId bursts are explained as radio emission from relativistic electron beams propagating nearly along the line of sight towards the observer with velocities close to the speed of light.

Acknowledgements. We are grateful to M. Poquérusse for useful discussion and comments. We thank K.-L. Klein and M.-P. Issartel for help with the processing and acces of NRH data. We are thankful to the TRACE, Yohkoh-SXT teams, Solar Data Analysis Center, and Mullard Space Science Laboratory for data access. Part of this work was supported by the German Deutsche Forschungsgemeinschaft, DFG MA 1376/14-1.

\section{References}

Alvarez, H., \& Haddock, F. T. 1973, Sol. Phys., 29, 197

Aschwanden, M. J., \& Acton, L. W. 2001, ApJ, 550, 475

Benz, A. O., Treumann, R., Vilmer, N., et al. 1982, A\&A, 108, 161

Bougeret, J.-L., Kaiser, M., Kellogg, P., et al. 1995, Space Sci., Rev., 71,231

Dulk, G. A., Goldman, M. V., Steinberg, J. L., \& Hoang, S. 1987, A\&A, 173, 366

Jiřička, K., Karlický, M., Kepka, O., \& Tlamicha, A. 1993, Sol. Phys., 147, 203

Karlický, M., \& Krlín, L. 1983, Bull. Astron. Inst. Czechosl. 34, 18

Karlický, M., \& Jungwirth, K. 1989, Sol. Phys. 124, 319

Karlický, M., Mann, G., \& Aurass, H. 1996, A\&A, 314, 303

Kerdraon, A., \& Delouis, J. M. 1997, in Lecture Notes in Physics 483, Coronal Physics from radio and space observations, ed. G. Trottet (Berlin: Springer), 192

Mann, G., Aurass, H., Voigt, W., \& Paschke, J. 1992, ESA-Journal SP-348, 129

Mann, G., Jansen, F., MacDowall, R. J., et al. 1999, A\&A, 348, 614

Melendez, J. L., Sawant, H. S., Fernandes, F. C. R., \& Benz, A. O. 1999, Sol. Phys., 187, 77

Melrose, D. B. 1980, Plasma astrophysics II (New York: Gordon \& Breach Publ.), 127

Pearson, T. J., \& Zensus, J. A. 1987, in Superluminal Radio Sources, ed. J. A. Zensus, \& T. J. Pearson (Cambridge Univ. Press), 1

Poquérusse, M. 1994, A\&A, 286, 611

Raoult, A., Lantos, P., Klein, K. L., Correia, E., \& Kaufmann, P. 1989, Sol. Phys., 120, 125

Sprangle, P., \& Vlahos, L. 1983, ApJ, 273, L95

Suzuki, S., \& Dulk, G. A. 1985, in Solar Radiophysics, ed. D. J. McLean, \& N. R. Labrum (Cambridge Univ. Press), 289

Wild, J. P. 1950, Aust. J. Sci. Res., Ser. A, 3, 541

Zlotnik, E. Ya., Klassen, A., Klein, K.-L., Aurass, H., \& Mann, G. 1998, A\&A, 331, 1087 